\title{
A numerical model to track two-fluid interfaces based on a stabilized finite element method and the level set technique
}

\author{
R. Codina ${ }^{1, *, \dagger}$ and O. Soto ${ }^{2, \ddagger}$ \\ ${ }^{1}$ Universitat Politècnica de Catalunya, Jordi Girona 1-3, Edifici C1, 08034 Barcelona, Spain \\ ${ }^{2}$ SCS/Laboratory for Computational Fluid Dynamics, George Mason University, MS 4C7, \\ 4400 University Drive, Fairfax, VA 22030-4444, USA
}

\begin{abstract}
SUMMARY
The objective of this work is to present a stabilized finite element formulation for transient incompressible flows and to apply it to the tracking of two-fluid interfaces. The stabilization technique employed allows us to use equal velocity-pressure interpolations and to deal with convection-dominated flows. The tracking of the fluid interface is based on the level set technique. A novel smoothing technique of this surface based on ideas of signal processing is presented. Copyright (c) 2002 John Wiley \& Sons, Ltd.
\end{abstract}

KEY WORDS: incompressible flows; stabilized finite element methods; level set; surface smoothing

\section{INTRODUCTION}

The purpose of this paper is two-fold. First, a stabilized finite element method to solve the transient incompressible Navier-Stokes equations is presented. Second, we describe a particular implementation of the so-called level set method to track interfaces between two fluids.

The numerical formulation presented here to solve the incompressible Navier-Stokes equations has three main features: the time discretization based on the standard trapezoidal rule, the stabilized finite element method and the iterative procedure, which in particular aims to uncouple the velocity-pressure calculation.

The important point of the numerical model is the stabilized finite element method we use. It is designed to allow both equal velocity-pressure interpolations (thus avoiding the need to satisfy the classical inf-sup condition) and to deal with convection-dominated flows, that is, situations in which the cell Reynolds number is greater than unity. This formulation, originally presented in References [1,2], is briefly described in the following section. It consists of

\footnotetext{
*Correspondence to: R. Codina, Universitat Politècnica de Catalunya, Jordi Girona 1-3, Edifici C1, 08034 Barcelona, Spain.

†E-mail: ramon.codina@upc.es, Web page: http://www.rmec.upc.es/homes/codina

$\ddagger$ E-mail: soto@scs.gmu.edu, Web page: http://www.scs.gmu.edu/ ${ }^{\sim}$ soto
}

Copyright (c) 2002 John Wiley \& Sons, Ltd.

Received May 2001

Revised September 2001 
adding to the Galerkin terms of the discrete variational equations a least-square form of the component of some terms orthogonal to the finite element space. These terms are those responsible for the instabilities of the Galerkin method, namely, the convective term and the pressure gradient.

Another important aspect of the numerical formulation is the iterative scheme. The iterations proposed here aim to deal with the non-linearity of the convective term, the projections involved in the stabilization terms, and also the uncoupling of the pressure from the momentum equation. This is particularly important from the computational standpoint, and needs to be treated carefully. Our scheme is motivated by the pressure segregation of some popular fractional step methods.

Referring to the evolution of the fluid interface, it is tracked using the so-called level set method (see References [3-5] for an overview), also called pseudo-concentration technique [6] and very similar to the volume of fluid (VOF) technique [7]. This formulation has been widely used to track free surfaces in mould filling (see for example References [8, 9], among other references) and other metal forming processes.

The level set method leads to a transport partial differential equation the solution of which determines the position of the free surface as an isovalue of the unknown of this equation, which we will call $\psi$. This equation is hyperbolic and therefore it is also necessary to use a stabilized finite element method to solve it. The important point in this case is that, even if the initial condition $\psi_{0}$ is a smooth function, if $\psi$ is maintained unmodified over several time steps it may begi Since the only impottan it is possible to smoth smoothing techniques novel smoothing algorit
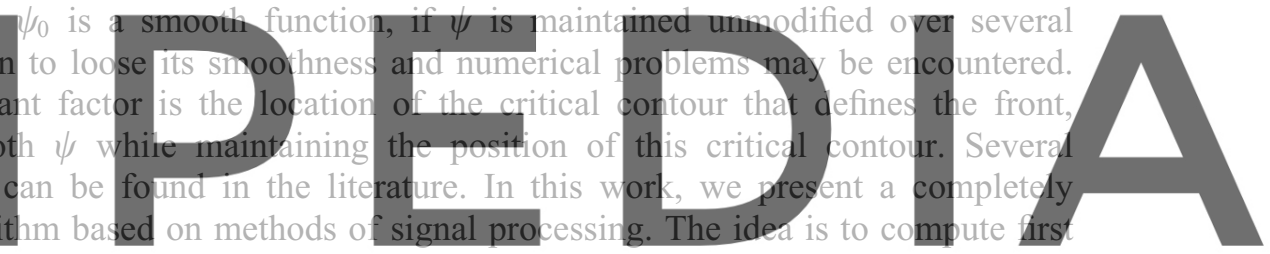

a 'noisy' distance function from each node to the fluid interface using a very fast algorithm.

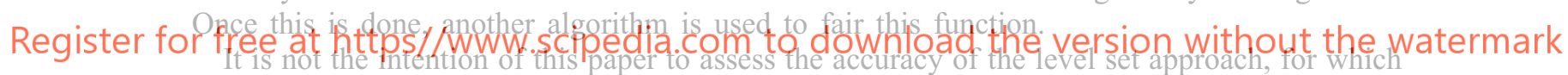

numerous references exist (see References [5, 10-12], where also a finite element formulation is used). The purpose of this article is to show how to use it with our stabilized finite element method and to demonstrate the effectiveness of the smoothing technique proposed here. This will be done in the numerical simulation of an industrial casting process.

\section{FINITE ELEMENT APPROXIMATION OF THE INCOMPRESSIBLE NAVIER-STOKES EQUATIONS}

The velocity and pressure fields of an incompressible fluid moving in the domain $\Omega$ during the time interval $\left(t_{0}, t_{f}\right)$ are governed by the incompressible Navier-Stokes equations

$$
\begin{array}{r}
\rho\left[\frac{\partial \boldsymbol{u}}{\partial t}+(\boldsymbol{u} \cdot \nabla) \boldsymbol{u}\right]-\nabla \cdot[2 \mu \boldsymbol{\varepsilon}(\boldsymbol{u})]+\nabla p=\boldsymbol{f} \\
\nabla \cdot \boldsymbol{u}=0
\end{array}
$$


where $\rho$ is the fluid density, $\boldsymbol{u}$ the velocity field, $\mu$ the dynamic viscosity, $\boldsymbol{\varepsilon}(\cdot)$ the symmetric gradient operator, $p$ the pressure and $f$ the vector external body forces, which includes the gravity force $\rho g$ and buoyancy forces, if required.

Let $\boldsymbol{\sigma}$ be the stress tensor and $\boldsymbol{n}$ the unit outward normal to the boundary $\partial \Omega$. Denoting by an over-bar prescribed values, the boundary conditions to be considered are:

$$
\boldsymbol{u}=\overline{\boldsymbol{u}} \text { on } \Gamma_{\mathrm{du}}, \boldsymbol{n} \cdot \boldsymbol{\sigma}=\mathbf{0} \text { on } \Gamma_{\mathrm{nu}}, \boldsymbol{u} \cdot \boldsymbol{n}=0, \boldsymbol{n} \cdot \boldsymbol{\sigma} \cdot \boldsymbol{g}_{1}=0, \boldsymbol{n} \cdot \boldsymbol{\sigma} \cdot \boldsymbol{g}_{2}=0 \text { on } \Gamma_{\mathrm{mu}}
$$

for $t \in\left(t_{0}, t_{f}\right)$. Vectors $\boldsymbol{g}_{1}$ and $\boldsymbol{g}_{2}$ (for the three-dimensional case) span the space tangent to $\Gamma_{\mathrm{mu}}$. Initial conditions have to be appended to the problem.

The condition on $\Gamma_{\mathrm{mu}}$ allows the fluid to slip on the boundary and is very often used for solid walls. Likewise, $\Gamma_{\mathrm{nu}}$ will be taken as the outflow boundary, where boundary conditions are in fact artificial, in the sense that the correct traction is unknown. On these boundaries we will also prescribe the pressure to zero, which together with $n \cdot \sigma=0$ implies that we consider negligible the normal component of the velocity gradients.

The stabilized formulation that will be used is a monolithic version of the orthogonal subscale stabilization method, hereafter referred to as OSS [1,2]. The original motivation of this method is that it allows to circumvent the well-known div-stability restriction for the velocity and pressure finite element spaces [13], allowing in particular equal interpolation for both unknowns. Likewise, it allows to deal with convection-dominated flows, eliminating the

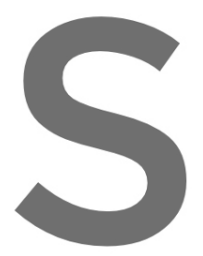
instability problems encountered when ziscous (or diffusive) te
Let $V_{h}^{*}$ and $Q_{h}^{*}$ be the finite element spaces to interpolat
respectively, constructed in the usual manner and using the
spaces one can construct the subspaces $J_{h, u}$ and $Q_{h}$ for the vel
tively. The former incorporates the Dirichlet conditions for the latter has one pressure fixed zero if the normal comp
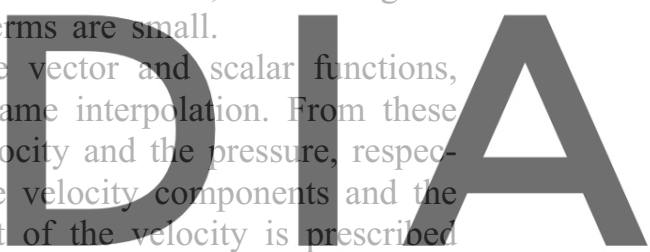

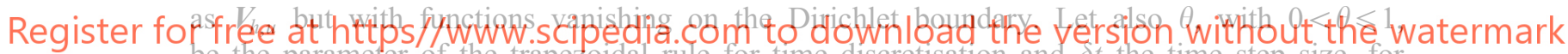

on the whole boundary. The space of velocity test functions, denoted by $V_{h}$, is constructed

be the parameter of the trapezoidal rule for time discretisation and $\delta t$ the time step size, for simplicity constant. The algorithmic solution to the problem will be computed at $t^{n}=n \delta t$, $n=1,2, \ldots$. The OSS monolithic discrete problem associated with the Navier-Stokes equations (1) and (2), discretizing in time using the generalized trapezoidal rule, and linearizing the convective term using a Picard scheme, can be written as follows: Given a velocity $\boldsymbol{u}_{h}^{n}$ at time $t^{n}$ and a guess for the unknowns at an iteration $i-1$ at time $t^{n+1}$, find $\boldsymbol{u}_{h}^{n+\theta, i} \in \boldsymbol{V}_{h, u}, p_{h}^{n+1, i} \in Q_{h}$, $\boldsymbol{\xi}_{u}^{i} \in \boldsymbol{V}_{h}^{*}, \boldsymbol{\xi}_{p}^{i} \in \boldsymbol{V}_{h}^{*}$ and $\xi_{d}^{i} \in Q_{h}^{*}$, by solving the discrete variational problem:

$$
\begin{aligned}
& \int_{\Omega} \rho \frac{\boldsymbol{u}_{h}^{n+\theta, i}-\boldsymbol{u}_{h}^{n}}{\theta \delta t} \cdot \boldsymbol{v}_{h} \mathrm{~d} \Omega+\int_{\Omega} \rho\left(\boldsymbol{u}_{h}^{n+\theta, i-1} \cdot \nabla\right) \boldsymbol{u}_{h}^{n+\theta, i} \cdot \boldsymbol{v}_{h} \mathrm{~d} \Omega \\
& \quad+\int_{\Omega} \mu \boldsymbol{\varepsilon}\left(\boldsymbol{u}_{h}^{n+\theta, i}\right): \boldsymbol{\varepsilon}\left(\boldsymbol{v}_{h}\right) \mathrm{d} \Omega-\int_{\Omega} \nabla \cdot \boldsymbol{v}_{h} p_{h}^{n+1, i-1} \mathrm{~d} \Omega-\int_{\Omega} \boldsymbol{v}_{h} \cdot \boldsymbol{f} \mathrm{d} \Omega \\
& \quad+\int_{\Omega} \tau_{1} \rho\left(\boldsymbol{u}_{h}^{n+\theta, i-1} \cdot \nabla\right) \boldsymbol{v}_{h} \cdot\left[\left(\boldsymbol{u}_{h}^{n+\theta, i-1} \cdot \nabla\right) \boldsymbol{u}_{h}^{n+\theta, i}-\boldsymbol{\xi}_{u}^{i-1}\right] \mathrm{d} \Omega \\
& \quad+\int_{\Omega} \tau_{2}\left(\nabla \cdot \boldsymbol{v}_{h}\right)\left(\nabla \cdot \boldsymbol{u}_{h}^{n+\theta, i}-\xi_{d}^{i-1}\right) \mathrm{d} \Omega=\mathbf{0}, \quad \forall \boldsymbol{v}_{h} \in \boldsymbol{V}_{h}
\end{aligned}
$$




$$
\begin{aligned}
& \int_{\Omega} \rho q_{h} \nabla \cdot \boldsymbol{u}_{h}^{n+1, i} \mathrm{~d} \Omega \int_{\Omega} \delta t \nabla q_{h} \cdot\left(\nabla p^{n+1, i}-\nabla p^{n+1, i-1}\right) \mathrm{d} \Omega \\
&+\int_{\Omega} \tau_{1} \nabla q_{h} \cdot\left(\nabla p^{n+1, i}-\boldsymbol{\xi}_{p}^{i-1}\right) \mathrm{d} \Omega=0, \quad \forall q_{h} \in Q_{h} \\
& \int_{\Omega} \boldsymbol{v}_{h}^{*} \cdot \boldsymbol{\xi}_{u}^{i} \mathrm{~d} \Omega=\int_{\Omega} \boldsymbol{v}_{h}^{*} \cdot\left(\boldsymbol{u}^{n+\theta, i} \cdot \nabla\right) \boldsymbol{u}_{h}^{n+\theta, i} \mathrm{~d} \Omega, \quad \forall \boldsymbol{v}_{h}^{*} \in \boldsymbol{V}_{h}^{*} \\
& \int_{\Omega} \boldsymbol{v}_{h}^{*} \cdot \xi_{p}^{i} \mathrm{~d} \Omega=\int_{\Omega} \boldsymbol{v}_{h}^{*} \cdot \nabla p^{n+1, i} \mathrm{~d} \Omega, \quad \forall \boldsymbol{v}_{h}^{*} \in \boldsymbol{V}_{h}^{*} \\
& \int_{\Omega} q_{h}^{*} \xi_{d}^{i} \mathrm{~d} \Omega=\int_{\Omega} q_{h}^{*} \nabla \cdot t_{h}^{n+\theta, i} \mathrm{~d} \Omega, \quad \forall q_{h}^{*} \in Q_{h}^{*}
\end{aligned}
$$

for $i=1,2, \ldots$ until convergence, that is to say, until $\boldsymbol{u}_{h}^{n+\theta, i-1} \approx \boldsymbol{u}_{h}^{n+\theta, i}$ and $p_{h}^{n+1, i} \approx p_{h}^{n+1, i-1}$ in the norm defined by the user. Subscript $h$ has been introduced to refer to the discrete finite element problem, while superscript $n$ refers to time $t^{n}$, and superscript $i$ to the iteration within the specific time step. In these equations, $\boldsymbol{u}_{h}$ and $p_{h}$ are approximations to the velocity and pressure fields, $\xi_{u}, \xi_{\mathrm{p}}$ and $\xi_{\mathrm{d}}$ the $L^{2}$ projections of the convective, pressure and divergence terms onto the finite element subspace, and $v_{h}, q_{h}, v_{h}^{*}$ and $q_{h}^{*}$ the test functions.

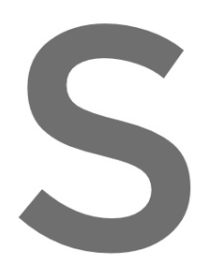

The first fiye tern The rest of terms ar stabilization of the streamline diffusion interpolation comes

A remark is needed in (3),
added to
a consist
for the the thion
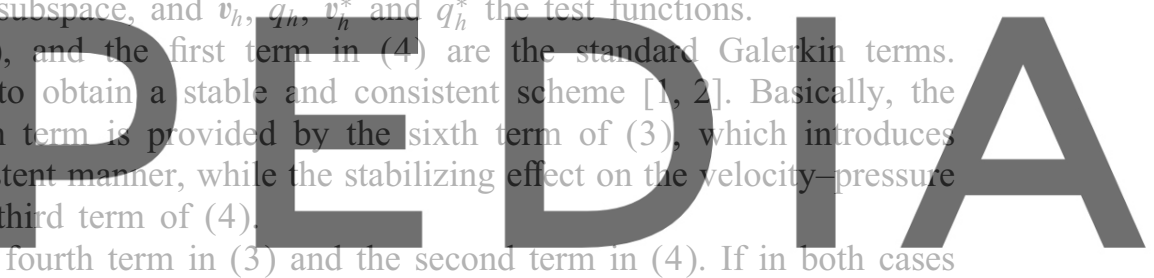

the pressure $p_{h}^{n+1, i-1}$ is replaced by $p_{h}^{n}$, we would have a second-order fractional step scheme,

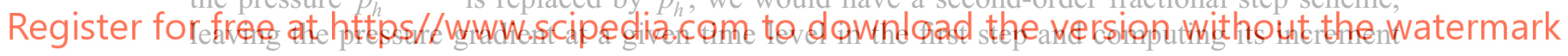

in the second one, with the discrete divergence of the pressure gradient approximated by the standard Laplacian (see References [14-16] for different ideas related to second-order fractional step schemes for the Navier-Stokes equations and Reference [17] for the stability analysis of this pressure treatment). Since we have used $p_{h}^{n+1, i-1}$ instead of $p_{h}^{n}$, our scheme can be viewed as a predictor-corrector method rather than a pressure splitting algorithm.

The parameters $\tau_{1}$ and $\tau_{2}$ are chosen in order to obtain a stable numerical scheme with optimal convergence rates (see References $[1,2,18,19]$ for details). They are computed within each element domain $\Omega^{e}$, with $e=1, \ldots, n_{\mathrm{el}}$, where $n_{\mathrm{el}}$ is the number of elements of the finite element partition. We take them as [19]

$$
\tau_{1}=\frac{\rho\left(h^{e}\right)^{2}}{4 \mu+2 \rho h^{e}\left|\boldsymbol{u}^{e}\right|} \quad \text { and } \quad \tau_{2}=\mu+\frac{1}{2} \rho h^{e}\left|\boldsymbol{u}^{e}\right|
$$

where $h^{e}$ and $\left|\boldsymbol{u}^{e}\right|$ are a typical length and a velocity norm of element $e$, respectively.

Once the algorithm has produced a converged solution, the velocity field at $t^{n+1}$ can be updated from the velocity at $t^{n+\theta}$ by using the relation $\boldsymbol{u}^{n+1}=\left[\boldsymbol{u}^{n+\theta}-(1-\theta) \boldsymbol{u}^{n}\right] / \theta$.

The accuracy of this formulation in the treatment of convection and pressure stabilization is discussed in Reference [1]. Likewise, the implementation and accuracy of the pressure stabilization is thoroughly discussed in Reference [20]. 


\section{IMPLEMENTATION OF THE LEVEL SET METHOD}

The basic idea of the level set method is to define a scalar function, say $\psi(\boldsymbol{x}, t)$, over the computational domain $\Omega$ in such a manner that its value at a certain point $\boldsymbol{x} \in \Omega$ indicates the presence or absence of fluid. This function may be considered as a fictitious fluid property which we will call pseudo-concentration. For instance, we may assign the value 1 to regions where fluid has already entered and the value of 0 to air-filled regions. The position of the fluid front will be defined by the iso-value contour $\psi(x, t)=\psi_{\mathrm{c}}$, where $\psi_{\mathrm{c}} \in[0,1]$ is a critical value defined a priori. The value $\psi_{\mathrm{c}}=0.5$ is usually taken. This value is immaterial if $\psi$ is a true step function, but is needed in the finite element discretisation and for the smoothing to be described later.

The conservation of the pseudo-concentration (assumed to be sufficiently smooth) in any control volume $V_{t} \subset \Omega$ which is moving with the divergence free velocity field $u$ leads to

$$
\frac{\partial \psi}{\partial t}+(\boldsymbol{u} \cdot \nabla) \psi=0 \quad \text { in } \Omega \times\left(t_{0}, t_{f}\right)
$$

This equation is hyperbolic and therefore boundary conditions for $\psi$ have to be specified at the inflow boundary, defined as
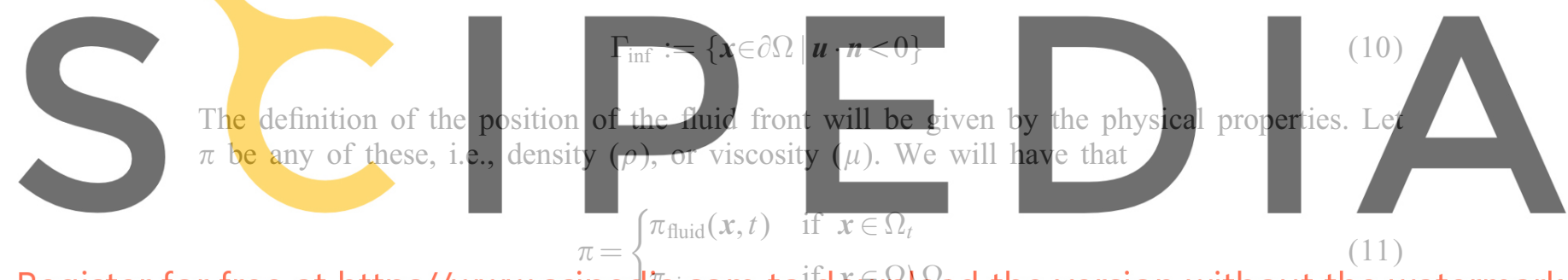

(11)

\section{Register for free at https//www.scipedia.com to ifbwhload the version without the watermark}

where $\Omega_{t}:=\left\{x \in \Omega \mid \psi(x, t) \geqslant \psi_{c}\right\}$, and the pseudo-concentration function $\psi$ is the solution of the following problem:

$$
\begin{array}{ll}
\frac{\partial \psi}{\partial t}+(\boldsymbol{u} \cdot \nabla) \psi=0 & \text { in } \Omega \times\left(t_{0}, t_{f}\right) \\
\psi=\bar{\psi} & \text { on } \Gamma_{\text {inf }} \times\left(t_{0}, t_{f}\right) \\
\psi(\boldsymbol{x}, 0)=\psi_{0}(\boldsymbol{x}) & \text { in } \Omega
\end{array}
$$

The initial condition $\psi_{0}$ is chosen in order to define the initial position of the fluid front to be analyzed. The boundary condition $\bar{\psi}$ determines whether fluid enters or not through a certain point of the inflow boundary.

Due to the pure convective type of the equation for $\psi$, we use the SUPG technique for the spatial discretisation. Again, the temporal evolution is treated via the standard trapezoidal rule.

If $\psi$ is taken as a step function, as indicated before, numerical problems may be encountered when it is transported. It is known that small oscillations in the vicinity of sharp gradients still remain using the SUPG formulation. These oscillations may propagate and yield to distorted 
front shapes, specially near corners. Since the basic idea of the method does not depend on the choice of the function $\psi$, it is preferable to use a smooth function instead of one with abrupt changes [8]. This can be achieved by redefining the pseudo-concentration for each node of the finite element mesh according to the following expression:

$$
\psi=\psi_{\mathrm{c}}+\operatorname{sgn}\left(\psi_{0}-\psi_{\mathrm{c}}\right) f(d)
$$

where $\psi_{0}$ stands for the calculated value of $\psi, f(d)$ is a function proportional to the distance (d) from the node under consideration to the front, and $\operatorname{sgn}(\cdot)$ is the signum of the value enclosed in the parenthesis.

The crucial point is how to calculate the distance $d$ from a point under consideration to the front. A totally new smoothing algorithm has been developed to compute $f(d)$ in (12). It is only based on the mesh topology, and on an approach to fair surfaces given in Reference [21]. To explain this technique, let us introduce first the concept of nodal levels.

Given a pseudo-concentration distribution, and therefore a front position, at each grid point $i$ of the mesh one may assign a level $l_{i}$ based on the following rules:

(i) A nodal point $i$ belongs to the level $l_{i}=0$, if it is filled with real fluid $\left(\psi_{i}>\psi_{\mathrm{c}}\right)$, but it is connected to at least one node $j$ filled with air $\left(\psi_{j}<\psi_{\mathrm{c}}\right)$.

(ii) A nodal point $i$ belongs to a level $l_{i}>0$, if it is filled with air $\left(\psi_{i}<\psi_{\mathrm{c}}\right)$, and it is

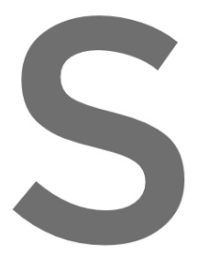
(iii) A nodal point $i$ belongs to a leve $l_{i}<0$, if it is flled with real flut $\left(\psi_{i}>\psi_{c}\right)$, and it
is connected to at least one node of level $l_{i}+1$.
The algorithm to assign the levels is very fast, and it only needs the pseudo-concentration
field and the arrays defining the mesh graph (the list of points surrounding each point).
Observe that the levels defined through this algorithm are integers.

Once the levels have been assigned to the mesh points, the distances $d_{i}$ from the points Register for free tathet computation is very fast if for each plane $p$ only the points surrounding the element in which $p$ is contained are checked. It is clear that the points belonging to levels 0 and 1 are the points that belong to the elements cut by the front. Then, $f(d)$ for a given node $i$ is defined by the following expression:

$$
f_{i}= \begin{cases}d_{i} & \text { if } 0 \leqslant l_{i} \leqslant 1 \\ d_{m}+\left(l_{i}-1\right) \bar{d} & \text { if } l_{i}>1 \\ d_{m}-l_{i} \bar{d} & \text { if } l_{i}<0\end{cases}
$$

where

$$
\bar{d}=\frac{1}{N} \sum_{i} d_{i} \quad \text { and } \quad d_{m}=\max _{i}\left\{d_{i}\right\} \quad \forall i \text { such that } 0 \leqslant l_{i} \leqslant 1
$$

and $N$ is the number of nodes belonging to levels 0 and 1 . Basically, the above equations define a scalar field that is proportional to $l_{i}$, and, therefore, that follows the mesh topology. However, such field has to be smoothed in some way to maintain its contour positions. Hence, an algorithm presented in Reference [21], and used to smooth surfaces without modifying the volume they enclose, was implemented. The method consists in redefining the smoothed 

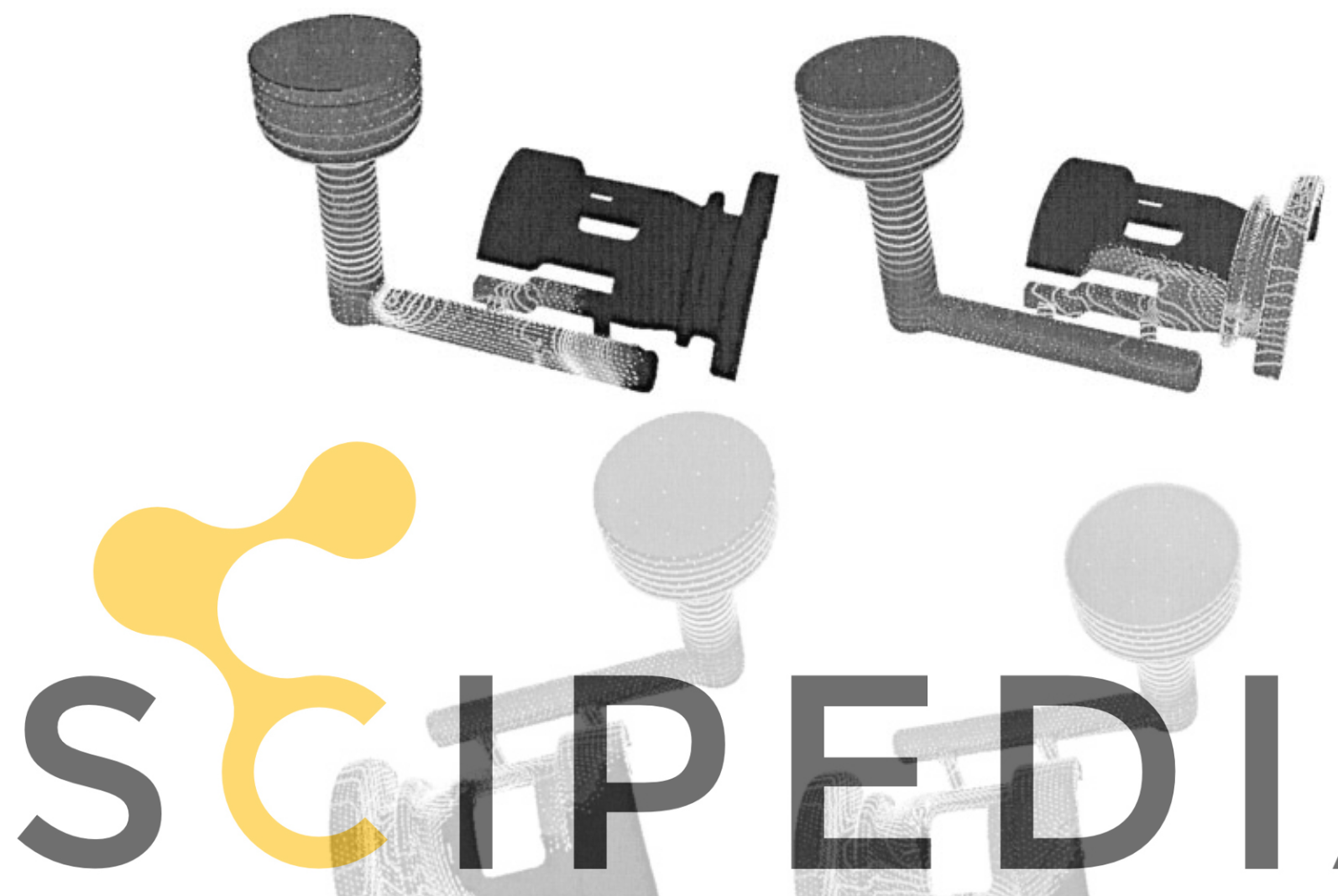

Figure 1. Position of the front of molten metal and pressure contours at $t=18$ (top left), $t=26$ (top right), $t=37$ (bottom left) and $t=40$ (bottom right).

function $f(d)$ for each nodal point that belongs to levels $l_{i}<0$ and $l_{i}>1$ as

$$
\begin{aligned}
f_{i}^{n-1 / 2} & :=f_{i}^{n-1}+\lambda \delta f_{i}^{n-1} \\
f_{i}^{n} & :=f_{i}^{n-1 / 2}+\mu \delta f_{i}^{n-1 / 2} \text { for } n=1, \ldots, n_{\text {pass }}
\end{aligned}
$$

where $n_{\text {pass }}$ is the number of smoothing passes, $\lambda$ and $\mu$ are constants with optimal values of 0.6313983 and -0.6739515 , respectively (see Reference [21]), and

$$
\delta f_{i}=\sum_{j \in \mathscr{N}_{i}} w_{i j}\left(f_{j}-f_{i}\right), \quad w_{i j}=\left(d_{i j} \sum_{j \in \mathscr{N}_{i}} \frac{1}{d_{i j}}\right)^{-1}
$$


where $\mathscr{N}_{i}$ is the neighbourhood of node $i$, that is, the set of nodes $j$ connected to the node $i$, and $d_{i j}$ is the distance between nodes $i$ and $j$.

Finally, the smoothed pseudo-concentration is defined by (12), taking $f(d)$ for the nodal point $i$ equal to $f_{i}^{n_{\text {pass }}}$. The number of passes to smooth $f$ was designed in a heuristic manner as $n_{\text {pass }}=\sqrt{n_{\mathrm{p}} / 2}$, where $n_{\mathrm{p}}$ is the total number of grid points. As a final remark, note that $f_{i}$ for the points of the levels $l_{i}=0$ and 1 is not smoothed to avoid variations in the free surface position; $f_{i}=d_{i}$ for such points. In fact, the smoothing could be applied only to the points neighbouring the free surface, and not to all the levels.

\section{NUMERICAL EXAMPLE}

In this section, we present the numerical simulation of the filling of a mould. In this case, the two fluids involved are molten metal and air. The finite element mesh employed in the analysis consists of approximately 40000 linear tetrahedra. The molten metal occupies a reservoir at the top of which the pressure is constant, and falls down to the mould through a pipe due to gravity. The material properties for the molten metal used in this example are (SI units) $\rho=6700$ and $\mu=10^{-3}$.

The results of the simulation are shown in Figure 1. The magenta colour corresponds to

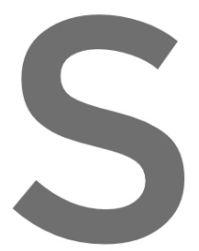

the molten material,

be observed there, the
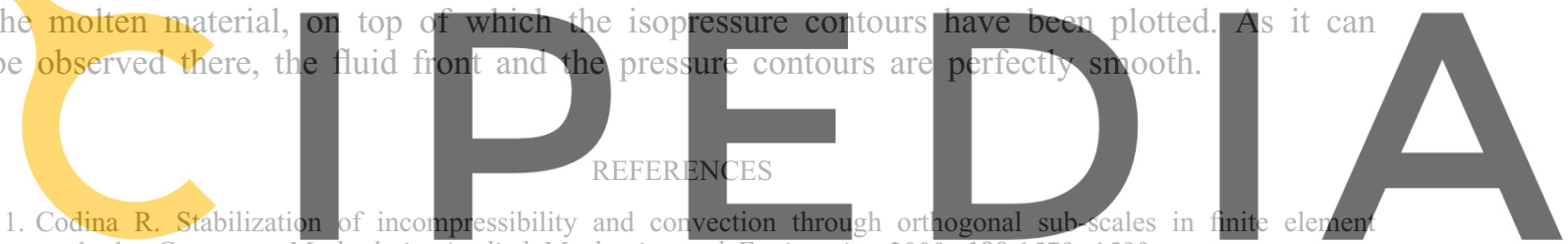

methods. Computer Methods in Applied Mechanics and Engineering 2000; 190:1579-1599.

2. Codina R. Stabilized finite element approximation of transient incompressible flows using orthogonal subscales. Journal of Computational Physics 1996; 124:449-464.

4. Sussman M, Almgren AS, Colella JB, Howell LH, Welcome ML. An adaptive level set approach for incompressible two phase flows. Journal of Computational Physics 1999; 148:81-124.

5. Osher S, Fedkiw RP. Level set methods: and overview and some recent results. Journal of Computational Physics 2001; 169:463-502.

6. Thompson E. Use of the pseudo-concentration to follow creeping viscous during transient analysis. International Journal for Numerical Methods in Engineering 1986; 6:749-761.

7. Hirt CW, Nichols BD. Volume of fluid (VOF) method for the dynamics of free boundaries. Journal of Computational Physics 1981; 39:201-225.

8. Codina R, Schäfer U, Oñate E. Mould filling simulation using finite elements. International Journal of Numerical Methods for Heat and Fluid Flow 1994; 4:291-310.

9. Lewis RW, Usmani AS, Cross JT. Efficient mould filling simulation in metal castings by an explicit finite element method. International Journal for Numerical Methods in Engineering 1995; 20:493-506.

10. Iafrati A, Di Mascio A, Campana EF. A level set technique applied to unsteady free surface flows. International Journal for Numerical Methods in Fluids 2001; 35:281-297.

11. Pichelin E, Coupez T. A Taylor discontinuous Galerkin method for the thermal solution in $3 \mathrm{~d}$ mold filling. Computer Methods in Applied Mechanics and Engineering 1999; 178:153-169.

12. Ilinca F, Hétu JF. Finite element solution of three-dimensional turbulent flows applied to mould-filling problems. International Journal for Numerical Methods in Fluids 2000; 34:729-750.

13. Brezzi F, Fortin M. Mixed and Hybrid Finite Element Methods. Springer: Berlin, 1991.

14. Bell JB, Colella P, Glaz HM. A second-order projection method for the incompressible Navier-Stokes equations. Journal of Computational Physics 1989; 85:257-283.

15. Shen J. On error estimates for some higher order projection and penalty-projection methods for Navier-Stokes equations. Numerische Mathematik 1992; 62:49-73. 
16. van Kan J. A second-order accurate pressure correction scheme for viscous incompressible flow. SIAM Journal on Scientific and Statistical Computing 1986; 7:870-891.

17. Codina R. Pressure stability in fractional step finite element methods for incompressible flows. Journal of Computational Physics 2001; 170:112-140.

18. Franca LP, Frey SL. Stabilized finite element methods: II. The incompressible Navier-Stokes equations. Computer Methods in Applied Mechanics and Engineering 1992; 99:209-233.

19. Codina R. A stabilized finite element method for generalized stationary incompressible flows. Computer Methods in Applied Mechanics and Engineering 2001; 190:2681-2706.

20. Codina R, Blasco J, Buscaglia GC, Huerta A. Implementation of a stabilized finite element formulation for the incompressible Navier-Stokes equations based on a pressure gradient projection. International Journal for Numerical Methods in Fluids 2001; 37:419-444.

21. Taubin G. A signal processing approach to fair surface design. IBM Research Report, 1995.
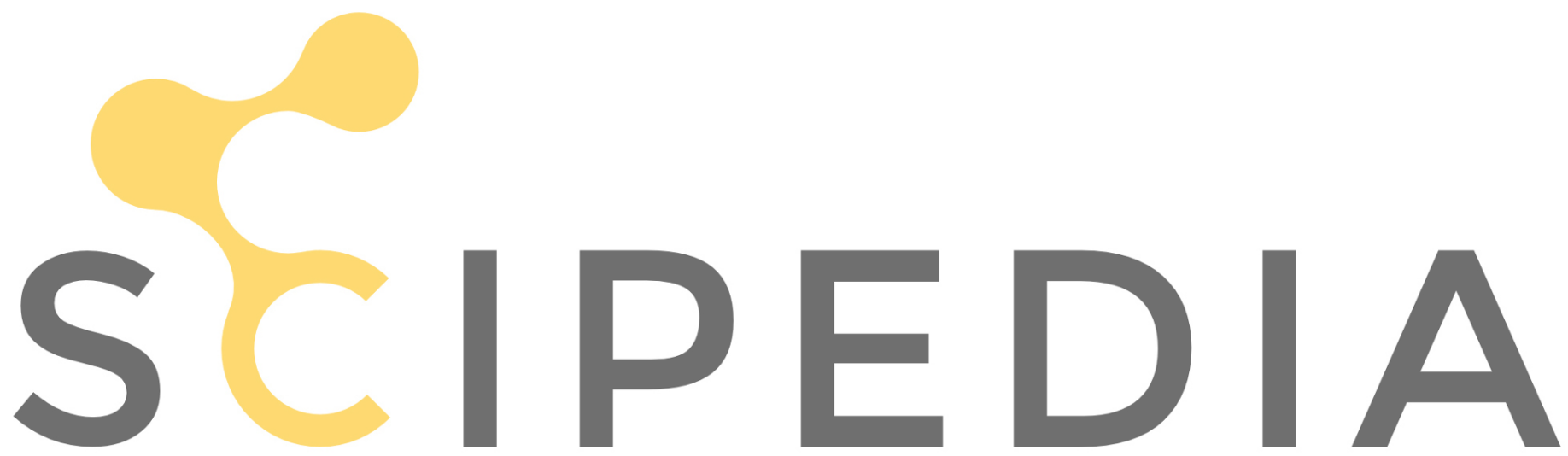

Register for free at https//www.scipedia.com to download the version without the watermark 